\title{
The impact of pneumococcal vaccination on pneumonia mortality among the elderly in Japan: A difference-in- difference study
}

\author{
Sung-mok Jung ${ }^{1}{ }^{,}$Hyojung Lee ${ }^{1}{ }^{\text {, Hiroshi Nishiura }}{ }^{\text {Corresp. }}{ }^{2}$ \\ ${ }^{1}$ Graduate School of Medicine, Hokkaido University, Sapporo, Hokkaido, Japan \\ 2 Graduate School of Medicine, Hokkaido University \\ Corresponding Author: Hiroshi Nishiura \\ Email address: nishiurah@gmail.com
}

Background. It is plausible that the routine immunization among infants using pneumococcal conjugate vaccine 13 (PCV13) from 2013 and among the elderly using pneumococcal polysaccharide vaccine 23 (PPV23) from 2014 contributed to reducing the pneumonia mortality among the elderly in Japan. The present study aimed to estimate the causal effect of this vaccination on pneumonia mortality, using the available cause-of-death data and employing a difference-in-difference (DID) design.

Methods. Two types of mortality data, i.e., prefecture-dependent and age- and gender-specific mortality data, from 2003-2017 were retrieved. We used mortality due to malignant neoplasm and heart disease as control groups and employed a DID design with an assumed parallel mortality trend between pneumonia and control group mortality since 2013 to estimate the causal effect of pneumococcal vaccination from 2014.

Results. Our estimation based on malignant neoplasm and heart disease as controls indicated that the reduced pneumonia mortality in 2017 owing to pneumococcal vaccination was as large as 41.9 (33.2, $50.6)$ and $31.2(23.8,38.6)$ per 100,000 individuals, respectively. The largest mortality reduction was observed for the oldest group (aged $\geq 90$ years), especially among men.

Discussion. The pneumococcal vaccination program, perhaps mainly represented by high vaccination coverage of PCV13 among children and partly by PPV23 administration with low coverage among the elderly in Japan, was shown to have reduced the pneumonia mortality in the elderly at the population level. 


\section{The impact of pneumococcal vaccination on pneumonia}

2 mortality among the elderly in Japan: A difference-in-

\section{3 difference study}

4

5 Sung-mok Jung ${ }^{1,2}$, Hyojung Lee ${ }^{1,2}$, Hiroshi Nishiura ${ }^{1,2 \S}$

6

7 1. Graduate School of Medicine, Hokkaido University, Sapporo, Japan

8 2. CREST, Japan Science and Technology Agency, Saitama, Japan

9

10 Corresponding Author:

11 Hiroshi Nishiura

12 Graduate School of Medicine, Hokkaido University, Sapporo, Japan

13 Kita 15 Jo Nishi 7 Chome, Kita-ku, Sapporo-shi, Hokkaido 060-8638, Japan

14 Email address: nishiurah@med.hokudai.ac.jp 


\section{Abstract}

17 Background. It is plausible that the routine immunization among infants using pneumococcal conjugate vaccine 13 (PCV13) from 2013 and among the elderly using pneumococcal polysaccharide vaccine 23 (PPV23) from 2014 contributed to reducing the pneumonia mortality among the elderly in Japan. The present study aimed to estimate the causal effect of this

21 vaccination on pneumonia mortality, using the available cause-of-death data and employing a

22 difference-in-difference (DID) design.

23 Methods. Two types of mortality data, i.e., prefecture-dependent and age- and gender-specific 24 mortality data, from 2003-2017 were retrieved. We used mortality due to malignant neoplasm 25 and heart disease as control groups and employed a DID design with an assumed parallel 26 mortality trend between pneumonia and control group mortality since 2013 to estimate the 27 causal effect of pneumococcal vaccination from 2014.

Results. Our estimation based on malignant neoplasm and heart disease as controls indicated that the reduced pneumonia mortality in 2017 owing to pneumococcal vaccination was as large as $41.9(33.2,50.6)$ and $31.2(23.8,38.6)$ per 100,000 individuals, respectively. The largest mortality reduction was observed for the oldest group (aged $\geq 90$ years), especially among men.

Discussion. The pneumococcal vaccination program, perhaps mainly represented by high vaccination coverage of PCV13 among children and partly by PPV23 administration with low coverage among the elderly in Japan, was shown to have reduced the pneumonia mortality in the elderly at the population level. 


\section{Introduction}

38 Japan, as a superaged nation, has a unique structure of causes of death (Arai et al., 2015). After

39 successfully controlling infectious diseases following World War II, the country experienced a

40 so-called "epidemiologic transition" with a dramatic change in the disease and cause-of-death

41 structures (Omran, 2005). In the recent decade, the four major causes of death have been

42 malignant neoplasm, heart disease, cerebrovascular disease (CVD), and pneumonia, all of which

43 are most frequently seen in the elderly (Ministry of Health, Labour and Welfare, 2018a) (Fig.

44 1A). While the CVD mortality has followed a declining trend, perhaps reflecting gradual

45 improvements in the diagnosis, treatment, and management of patients, the mortality from the

46 other three major causes has steadily increased over time (Fig. 1B). These increases are

47 considered to reflect the aging process as a whole in addition to aging within the elderly group.

48 Unfortunately, from a clinical point of view, there has been no breakthrough in the prevention or

49 treatment for these three diseases (Ebihara, 2017).

50 Nevertheless, it is noteworthy that an important change has occurred in reducing the risk

51 of community-acquired pneumonia, especially pneumococcal pneumonia caused by

52 Streptococcus pneumoniae, which accounts for $18.8 \%$ of the total cases (The Japanese

53 Respiratory Society, 2017; Miyashita \& Yamauchi, 2018). Following the subsidy-based

54 exploratory immunization that began in 2009 at a municipality level with small coverages, in

552014 Japan began a routine immunization program with the pneumococcal polysaccharide

56 vaccine 23 (PPV23) among the elderly (Naito, Yokokawa \& Watanabe, 2018). In that program,

57 elderly individuals aged 65 years or older were eligible for vaccination; owing to the policy

58 intent to cover the entire elderly population within 5 years, those who newly reached the age of

$5965,70,75,80,85,90,95$, or 100 years from $2014-2018$ were invited to receive the 
60 immunization (Naito, Yokokawa \& Watanabe, 2018). At yearly basis, crude vaccination

61 coverage of PPV23 among elderly from 2014-16 was estimated at 38.3\%, 33.5\% and 37.8\%,

62 respectively (Ministry of Health, Labour and Welfare, 2018b). As of 2018, a vaccination

63 coverage slightly above $40 \%$ has been achieved among the elderly (Naito, Yokokawa \&

64 Watanabe, 2018; Ministry of Health, Labour and Welfare, 2018b). In addition to vaccinating the

65 elderly with PPV23, which is considered to protect vaccinated individuals from 23 types of

66 pneumococci bacteria, the immunization of infants with the pneumococcal conjugate vaccine

67 (PCV)7 was introduced as voluntary vaccination from late 2010 with the initially estimated

68 coverage below 40\%. Subsequently, public subsidy of PCV7 vaccination started in 2011 or 2012

69 depending on policy decision by local governments, and where subsidized, the vaccination

70 coverage was elevated to be greater than $80 \%$ in the corresponding cities. In 2013 , PCV13

71 replaced PCV7, and the vaccination with PCV13 became a part of routine immunization

72 program. Regardless of PCV7 or PCV13, the first, second and third doses were given at 2

73 months old, 4 months old and 6 month old, respectively, with an optional supplementary dose at

74 12-15 months old. Yearly vaccination coverage among children for three doses of PCV13, as

75 calculated by the ratio of the total number of third doses to the demographic size of the subject

76 age group, from $2013-16$ all exceeded $99.9 \%$ (i.e. $103.4 \%, 102.5 \%, 106.5 \%$ and $99.9 \%$,

77 respectively), because people receiving this vaccination may have slightly extended to other

78 (perhaps older) age groups (Ministry of Health, Labour and Welfare, 2018b). Considering the

79 high vaccination coverage, this change has been expected to not only reduce the invasive

80 pneumococcal disease among infants but also reduce the transmission of these diseases from

81 children to the elderly (Bogaert, De Groot \& Hermans, 2004). 
83 the pneumonia mortality among the elderly in Japan. A published study in Canada indicated that

84 the risk of death, frequency of complications, and length of hospital stay among pneumonia

85 patients were all reduced by prior pneumococcal vaccination (Fisman et al., 2006). Moreover,

86 PPV23 has been shown to prevent pneumonia in elderly people (Ortqvist et al., 1998).

87 Nevertheless, a population-based cohort study involving hospital-admitted pneumonia patients in

88 Canada demonstrated that, if admitted patients were vaccinated and followed up, PPV23

89 immunization was not associated with a reduced risk of death or further hospitalization

90 (Johnstone et al., 2010). Another cohort study in Italy has shown that while PPV23

91 administration certainly improved the survival rate of elderly individuals, those who received

92 PCV13 enjoyed a better survival rate (Baldo et al., 2016). Scientific evidence supporting the

93 effectiveness of pneumococcal vaccines for preventing pneumonia death is still scarce, and it has

94 not been directly assessed among the elderly in Japan.

95 A close look at the pneumonia mortality in Japan in recent years (Fig. 1B) reveals an

96 overall stagnation with a potential signature of decline. Notably, aging progressed during this

97 period both for the entire population and within the elderly population, and, in line with this, the

98 mortality due to two other major causes, i.e., malignant neoplasm and heart disease, steadily

99 increased from 2003-2017. This observation may offer an important avenue by which to study

100 the effectiveness of pneumococcal vaccination in Japan. The present study aimed to estimate the

101 impact of pneumococcal vaccination on pneumonia mortality among the elderly, using the cause-

102 of-death data in Japan and employing a quasi-experimental study design. 


\section{Materials \& Methods}

\section{Mortality data}

105 The present study was conducted using publicly available datasets on the cause-of-death in Japan

106 from 2003-2017 (Ministry of Health, Labour and Welfare, Japan, 2018a). The time frame of

107 2003-2017 was specifically selected because the elderly aged 80 years or older were grouped

108 together in the data from earlier years and also because the age-specific mortality trend appeared

109 to be approximately linear during this period based on a visual assessment. Otherwise, the data

110 were available in five-year age bands. We restricted ourselves to analyzing the mortality trends

111 of only three major causes of death, namely, malignant neoplasm, heart disease, and pneumonia,

112 because these were expected to increase with the aging population and there have not been any

113 breakthroughs other than pneumococcal vaccination that might abruptly reduce these mortalities.

114 We collected two types of mortality data. One was the prefecture-dependent mortality

115 rate per 100,000 individuals from 2003-2017. For this, the cause-specific mortality rates for 47

116 prefectures were amassed. The other dataset was the age- and gender-dependent mortality rate

117 per 100,000 individuals from 2003-2017. Ages were grouped in 5-year increments. Among the

118 elderly, pneumonia was ranked within the top five causes of death from around the ages of 65-

11969 years old. Thus, our subject ages were split into six age-groups, i.e., those aged 65-69, 70-74,

$12075-79,80-84,85-89$, and $\geq 90$ years, stratified by gender. Moreover, to validate our underlying

121 assumption of parallel trend between pneumonia mortality and control disease selected either

122 from malignant neoplasm or heart disease, we also collected the mortality data due to chronic

123 obstructive pulmonary disease (COPD) and used it as the control during sensitivity analysis (see

124 statistical analysis). To do so, the COPD-induced mortality data for the entire Japan from 2003-

12517 was collected, and compared against pneumonia mortality. 


\section{Epidemiological analysis}

127 The present study employed a difference-in-difference (DID) design, which is a quasi-

128 experimental epidemiological study design that can be applied to observational data (Wing,

129 Simon \& Bello-Gomez, 2018). To purify the causal impact of pneumococcal vaccination on

130 pneumonia mortality among the elderly, we compared the mortality trend of pneumonia death

131 against the mortality of two control cause-of-death groups, i.e., malignant neoplasm and heart

132 disease. From a visual assessment of the data shown in Figure 1B, the mortality of these three

133 major diseases have increased roughly in parallel over the last decades. Exploiting this

134 observation, we independently applied the DID design to both the prefecture and age/gender

135 data.

136 We used $g=1, \ldots, G$ to index cross-sectional units, such as geographic areas, $a=1, \ldots, A$

137 to index age groups, and $t=2003, \ldots, 2017$ to indicate year. The term $Y_{g t}^{k}$ represents the outcome

138 (i.e., mortality) against the mortality of control group $k$ for unit $g$ in year $t$. For the analysis of

139 prefectural data, we used the expected mortality value calculated as

$$
E\left(Y_{g t}^{k}\right)=\alpha_{0}+\alpha_{1}\left(t-t_{0}\right)+\beta\left(t-t_{v}\right)\left(T_{g}^{k} P_{t}\right)+\gamma T_{g}^{k}+\delta P_{t},(1)
$$

141 to predict the pneumonia mortality and also the mortality of control disease $k$, where $\alpha_{0}$ and $\alpha_{1}$

142 describe the mortality of a control group that is assumed to share the same mortality trend in

143 parallel with the pneumonia group over a set time period, $t_{0}$ is the first year of our analysis (i.e.,

$\left.144 t_{0}=2003\right), \beta$ is the causal effect of vaccination on pneumonia mortality, $t_{v}$ is the first year when

145 vaccination starts to influence the dynamics (i.e., $t_{v}=2013$ ), $\gamma$ is the group effect on pneumonia

146 mortality, and $\delta$ is the time effect on both pneumonia and control group $k$ that was introduced at

147 the same time as the pneumococcal vaccination. Because routine pneumococcal immunization

148 with PCV for infants began in 2013, $t_{v}$ was assumed to be 2013. Because Japan implemented 
149 vaccination spending 5 years to cover all corresponding birth cohorts of elderly, and due to

150 gradual roll-out effect of indirect benefit, the causal effect in the present study is modeled as the

151 rate of change per year (i.e. expressed as yearly reduction rate in pneumonia mortality). $T_{g}^{k}$ and $P_{t}$

152 are dichotomous dummy variables, defined as

$$
T_{g}^{k}=\left\{\begin{array}{l}
1 \text { for pneumonia group } \\
0 \text { for control group }
\end{array},\right.
$$

154 and

$$
P_{t}=\left\{\begin{array}{l}
1 \text { for } \mathrm{t} \geq 2014 \\
0 \text { otherwise }
\end{array}\right.
$$

156

respectively.

Similarly, but separately, we analyzed the dataset of age- and gender-dependent mortality data. The term $Y_{\text {sat }}^{k}$ represents the outcome (i.e., the nationwide mortality) of age $a$ and gender $s$ in year $t$ against the mortality of control group $k$. We used the expected mortality value calculated as

$$
E\left(Y_{s a t}^{k}\right)=\alpha_{0, s a}+\alpha_{1, s a}\left(t-t_{0}\right)+\beta_{s a}\left(t-t_{v}\right)\left(T_{g}^{k} P_{t}\right)+\gamma_{s a} T_{g}^{k}+\delta_{s a} P_{t},
$$

to predict the pneumonia mortality and also the mortality of a control disease, where $t_{0}, t_{v}$, and dummy variables $T_{g}^{k}$ and $P_{t}$ are the same as in equation (1). The difference between model (4) and model (1) is that the parameters, $\alpha_{0}, \alpha_{1}, \beta, \gamma$, and $\delta$ are all dealt with as a function of age $a$ and gender $s$ for control disease group $k$ in model (4).

\section{Statistical analysis}

For each control disease, i.e., malignant neoplasm and heart disease, we separately estimated the causal effect of pneumococcal vaccination $\beta$ on pneumonia mortality for both models (1) and (4). As part of sensitivity analysis, an additional analysis was conducted using the COPD as a control disease, and we confirmed that the causal impact of pneumococcal 
171 vaccination on pneumonia mortality among elderly would be maintained. The tenth revision of

172 the International Classification of Diseases (ICD-10) began to be strictly enforced in January

1732017 with respect to the rule to specify the underlying cause of death (rather than identifying the

174 resulting pathophysiological process as the cause of death) (Ministry of Health, Labour and

175 Welfare, 2018a), and thus, the time-dependent decline in pneumonia mortality may potentially

176 be explained by this change. To avoid misattributing that overlapping effect, we estimated the

177 causal effect of vaccination by analyzing the mortality both with and without the 2017 data. The

178 non-linear least sum of squares method was employed to fit models (1) and (4) to the

179 corresponding data from 2003-2017. The 95\% confidence intervals (CI) of parameters were

180 computed by the profile likelihood method. All statistical data were analyzed using JMP version

181 12.0.1 statistical software (SAS Institute Inc., Cary, NC, USA).

\section{Ethical considerations}

183 The present study analyzed data that is publicly available (Ministry of Health, Labour and

184 Welfare, 2018a). As such, the datasets used in our study were de-identified and fully anonymized

185 in advance, and the analysis of publicly available data without identity information does not

186 require ethical approval.

\section{Results}

188 Table 1 shows the summary statistics of mortality for three causes of death, i.e., pneumonia, 189 malignant neoplasm and heart disease. All of them showed increasing trend with age. The gap of

190 mortality by age was greatest for pneumonia, e.g. while the pneumonia mortality among those

191 aged 65-69 years were 43 and 11 per 100,000 for male and female, respectively, these figures

192 among people aged 90 years and older were 2,883 and 1,438 per 100,000, respectively. We have 
193 compared correlations of mortality between pneumonia and control disease (i.e. malignant

194 neoplasm or heart disease) by age group and gender (see Online Supplementary Table 1).

195 Comparing those correlations from 2003-13 and from 2014-17, we found that the parallel trend

196 between pneumonia and control disease was maintained better prior to 2013 , and subsequently

197 the correlations were mostly diminished. During the latter part of the time period, the pneumonia

198 mortality data were probably affected by vaccination.

199 The estimated risk reductions of pneumonia mortality attributable to pneumococcal

200 vaccination, using the mortality of malignant neoplasm or heart disease as the control, are shown

201 in Figure 2. In all analyses, the upper 95\% CI values were less than 0 , and a time-dependent

202 increase in the causal reduction effect of pneumococcal vaccination on pneumonia mortality was

203 observed. Table 2 shows estimates of the causal parameter $\beta$ which indicates the yearly reduction

204 rate of pneumonia mortality that can be attributed to pneumococcal vaccination (see Online

205 Supplementary Table 2 for other parameters). When the 2017 data were included, the analysis

206 with malignant neoplasm or heart disease as a control indicated that the reduced pneumonia

207 mortality in 2017 was as large as $41.9(33.2,50.6)$ or $31.2(23.8,38.6)$ per 100,000 individuals,

208 respectively. When the 2017 data were excluded from the analyses, these mortality reductions

209 did not deviate significantly and were estimated at $34.6(22.0,47.1)$ or $19.0(8.3,29.6)$ per

210100,000 when malignant neoplasm or heart disease, respectively, was used as the control.

211 Comparisons of the expected and observed mortality values are shown in Figure 3, and

212 parameter estimates of the causal model are shown as Table 3 . The observed values are

213 expressed as the distribution of mortality in the 47 prefectures (see Online Supplementary Table

2143 for the observed values of the entire Japan). Both control diseases had a pattern of monotonic

215 increase in the mortality over time for both the expected and observed mortality; they shared this 
216 increasing trend with pneumonia mortality through 2013. However, an abrupt decline in the

217 pneumonia mortality was observed beginning in 2013. This phenomenon was also captured by

218 the simple DID model described above; together, they support the causal effect of pneumococcal

219 vaccination on the decline in pneumonia mortality.

220 The estimated causal effects of pneumococcal vaccination in reducing age- and gender-

221 specific pneumonia mortality, using malignant neoplasm and heart disease as the control group

222 based on analyses with and without the 2017 data, are shown in Figure 4 . We did not identify

223 statistically significant reductions in the pneumonia mortality among those aged from 65 to 79

224 years for either males or females in any of our analyses. In the older age groups, vaccine-induced

225 reductions in the pneumonia mortality were seen, and the estimated effect size grew with the

226 subject age, especially among men. In the analyses that included the 2017 data and used

227 malignant neoplasm or heart disease as the control, the pneumonia mortality in elderly males

228 aged $\geq 90$ years was reduced every year from 2014 by 357.4 (95\% CI: $314.0,400.7)$ or 276.3

229 (95\% CI: 237.3, 315.2) per 100,000 individuals, respectively, as a function of time since

230 vaccination effect. Similarly, the yearly reduction in pneumonia mortality among females was

231 estimated at 185.5 (95\% CI: $156.2,214.9)$ or 105.2 (95\% CI: $70.2,140.3)$ per 100,000

232 individuals when malignant neoplasm or heart disease, respectively, were used as the control.

233 There was a good overall level of agreement between the observed and expected

234 mortality data with respect to age and gender for pneumonia (Fig. 5A, B, E, and F) and the

235 control causes of death (Fig. 5C, D, G, and H). In different age and gender groups, a different

236 linear trend of mortality was shared between pneumonia and each control disease beginning in

237 2013, after which a clear decline in pneumonia mortality was identifiable, especially among

238 those aged $\geq 90$ years. 
As part of sensitivity analysis, Table 4 examines the causal effect when 2014 was used,

240 rather than 2013, as the year of change point, considering that the completion of vaccination

241 among elderly is 2014 , while that of children was 2013 . The yearly reduction rate in pneumonia

242 mortality was estimated to be greater for this model, compared with 2013 model. Causal

243 parameter estimates in the 2014 model were greater than those of 2013 model, because

244 substantial number of elderly started to be vaccinated within 2013 and corresponding herd

245 immunity effect was perhaps captured by the 2014 model.

246 The mortality of COPD was used as the control as part of sensitivity analysis. As was

247 also the case for malignant neoplasm and heart disease, the overall parallel trend between COPD

248 and pneumonia mortality prior to vaccination, and an assumed causal impact of vaccination on

249 mortality were reflected in the prediction using model (1) and qualitatively captured the observed

250 patterns for the entire Japan (see Online Supplementary Figure 1). The maximum likelihood

251 estimate of causal parameter remained to be negative (Online Supplementary Table 4), i.e. -2

252 (95\% CI: -5, 1), not much deviated from the results using other control diseases (Table 3).

\section{Discussion}

254 The present study estimated the causal effect of pneumococcal vaccination on pneumonia

255 mortality among the elderly in Japan. A DID study design, which is a quasi-experimental

256 epidemiological research design, was employed, using the time-dependent mortality of malignant

257 neoplasm and heart disease as the control groups because these causes of death demonstrated a

258 monotonic increase induced by population aging and shared this trend with pneumonia mortality

259 at least through 2013. The subsequent abrupt decline in pneumonia mortality was captured

260 through the analysis of prefectural data as well as of age- and gender-specific nationwide data.

261 Overall, the pneumonia mortality in 2017 was considered to have been reduced by $20-40$ per 
262100,000 individuals owing to pneumococcal vaccination, with the largest causal effect occurring

263 among the oldest group, aged $\geq 90$ years. To our knowledge, the present study is the first to have

264 assessed the combined causal impact of two pneumococcal vaccination programs, i.e., PCV13

265 among children and PPV23 among the elderly, in reducing pneumonia mortality in the elderly as

266 evaluated at the population level. The vaccination coverage of PCV13 among children has

267 achieved over 95\%, while that among elderly with PPV23 has remained less than 50\%, implying

268 that the large population impact may be represented by herd immunity due to PCV13.

269 It is remarkable that an explicit mortality reduction was identified by this quasi-

270 experimental study design. Our analyses revealed that larger causal impacts of pneumococcal

271 vaccination on pneumonia mortality were observed in the more recent years. Although this

272 causal impact was not clearly identified among elderly individuals aged from 65-79 years, a

273 clear impact was seen for people in their 80s and 90s. The question of whether PPV23

274 administration results in a visible causal impact among elderly people has been controversial:

275 while protection against invasive pneumococcal disease was identified (Cadeddu et al., 2012)

276 and a certain protection against pneumococcal pneumonia was reported (Chidiac and Ader,

277 2009; Falkenhorst et al., 2017), the effectiveness of PPV23 was smaller than that of PCV13

278 (Baldo et al., 2016) and sometimes was only minimal (Johnstone et al., 2010). However, the

279 indirect effect of PCV13 vaccination in infants and children on outcomes in adults and the

280 elderly has been reported (Simonsen et al., 2014; Regev-Yochay et al., 2017; Tsaban and Ben-

281 Shimol, 2017); this practice of pediatric vaccination clearly provides a herd immunity effect

282 among the elderly (Haber et al., 2007; Simonsen et al., 2011). Although our study endorses those

283 findings in other countries including North America, because of the population-based design of

284 this work, we were not able to disentangle the causal effects attributed to the two vaccines. 
285 Considering limited coverage of PPV23 and high coverage of PCV13, it is likely that the

286 observed reduction in pneumonia mortality among elderly can be attributed to herd immunity

287 impact by PCV13. In fact, looking at the most vulnerable group aged 90 years and older (Figure

288 5A), the decline in pneumonia mortality has started to take place since subsidy among infants

289 from 2011, and the gradual decline afterwards is consistent with the gradual increase in

290 vaccinated children with PCV7 or PCV13. On the other hand, we do not identify visible impact

291 of PPV23 introduction from 2013. However, due to similar timing of introduction for both

292 vaccines, the observed effect can be still be partially attributed to not only PCV13 but also

293 PPV23, and the present study was not able to purify the unique effect of PCV13 alone from the

294 mixed population impact of PCV13 and PPV23.

295 On the basis of the nature of this study's DID design, other plausible explanations for the

296 time-dependent decline in pneumonia mortality were mostly excluded. Although the DID design

297 required the assumption of proportional trends among the pneumonia, malignant neoplasm, and

298 heart disease mortalities, the reasonable fits produced by these data justified the use of these

299 control diseases. Furthermore, the monotonic time-dependent increase was mechanistically

300 explainable based on progressive population aging. Because the 2017 data could have been

301 impacted by ICD-10's rule to identify the underlying cause of death, we conducted analyses both

302 with and without the 2017 data, but the overall qualitative patterns of our findings were not

303 altered by the exclusion. This finding supports our conclusion that the abrupt decline in

304 pneumonia mortality in 2017 is not fully explained by the ICD-10 change.

305 In Japan, the number of individuals who "died of old age" (i.e., natural death from an

306 ailment associated with aging) has steadily increased. It is possible that the category "died of old

307 age" absorbed the apparent reduction in pneumonia deaths in recent years. However, that 
308 possibility leaves the question of why only pneumonia deaths, not those due to neoplasm or heart

309 disease, were selectively miscategorized. Our use of malignant neoplasm and heart disease as

310 controls in the DID design helped to eliminate several concerns that the recent reductions in

311 pneumonia mortality could be attributed to causes other than the two pneumococcal vaccination

312 programs.

313 The present study using mortality as the outcome succeeded in demonstrating that even

314 this type of quasi-experimental study can offer a vivid effect size estimate of pneumococcal

315 vaccination programs at the population level. To truly estimate the individual-based causal effect

316 of vaccination, it is critical to conduct a prospective study. Such a study should ideally control

317 the exposure (i.e., frequency of contact) to children, so that the indirect effect of PCV13

318 vaccination may be separated from the direct benefit of PPV23 vaccination.

319 Five limitations of this study should be noted. First, control groups other than malignant

320 neoplasm and heart disease deaths were not explored. It is possible that a more monotonically

321 increasing control (e.g., mortality following bone fracture) could be conceived and compared

322 against pneumonia mortality; however, we had access to only major causes of death by

323 prefecture and by age and gender. Second, the cause of death category of pneumonia includes

324 not only community-acquired pneumonia but also hospital-acquired infections and aspiration

325 pneumonia. The observed reduction in pneumonia mortality was not verified to have been caused

326 by a specific reduction in mortality from pneumococcal pneumonia, and moreover, possible

327 improvement in prognosis could have been absorbed as our causal effect of vaccination.

328 Nevertheless, even provided that we explored more detailed etiology of pneumonia, the time

329 trend might not be easily explainable. Third, the effectiveness estimate may be quantitatively

330 associated with the vaccination coverage both in children and the elderly; thus, variations in the 
331 causal impact by prefecture could possibly be explained by differences in vaccination coverage.

332 Unfortunately, we were not able to conduct an analysis of this possibility owing to limitations

333 with our data. Fourth, PCV7 vaccination was introduced as voluntary vaccination in 2010, and

334 the subsidy-based program started in 2011 at the municipal level; either or both could have

335 affected the pneumonia mortality. However, coverage with the PCV7 vaccination remained very

336 low in 2014. Similarly, the overall vaccination coverage from the subsidy-based program

337 remained very low, not even reaching a few percent. Only in and after 2014 was the

338 pneumococcal vaccination coverage of both children and the elderly elevated to substantial

339 levels. Fifth, we did not have an access to the temporal datasets of invasive pneumococcal

340 disease (IPD) among children over time. It is fruitful to estimate the causal impact of

341 pneumococcal vaccination among children, looking into the corresponding data of IPD.

342 To confirm our findings, a precise estimation of the causal effect of pneumococcal

343 vaccination among the elderly must be determined by conducting a study with a prospective

344 design. Nevertheless, by using only the available census-based observational data, we showed

345 that a DID design can be exploited to examine the population impact of pneumococcal

346 vaccination on pneumonia mortality among the elderly. We believe that our approach will shed

347 light on the assessment of the combined effectiveness of pneumococcal vaccinations in Japan.

\section{Conclusions}

349 The present study employed a DID design using the time-dependent mortality of malignant

350 neoplasm and heart disease as control groups to estimate the causal effect of pneumococcal

351 vaccination on pneumonia mortality among the elderly in Japan. From 2014, an abrupt decline in

352 pneumonia mortality was seen; specifically, the mortality was reduced by $20-40$ per 100,000 
353 individuals because of pneumococcal vaccination, with the largest causal effect among the oldest

354 group aged 90 years and older.

\section{References}

356 Arai H, Ouchi Y, Toba K, Endo T, Shimokado K, Tsubota K, Matsuo S, Mori H, Yumura W,

357 Yokode M, Rakugi H, Ohshima S. 2015. Japan as the front-runner of super-aged societies. Perspectives from Medicine and Medical Care in Japan 15:673-687 DOI: 10.1111/ggi.12450.

Baldo V, Cocchio S, Gallo T, Furlan P, Romor P, Bertoncello C, Buja A, Baldovin T. 2016. Pneumococcal conjugated vaccine reduces the high mortality for community-acquired to pneumococcal disease. Lancet Infectious Diseases 4:144-154. pneumococcal polysaccharide vaccine (PPV23) for the prevention of invasive pneumococcal diseases (IPDs) in the elderly: is it really effective? Journal of Preventive Medicine and Hygiene 53:101-103.

Chidiac C, Ader F. 2009. Pneumococcal vaccine in the elderly: a useful but forgotten vaccine. Aging Clinical and Experimental Research 21:222-228.

Ebihara S. 2017. Up-to-date prevention of aspiration and pneumonia among elderly. Geriatric Medicine 55:1183-1184. 
against pneumococcal disease in the elderly: systematic review and meta-analysis. PLoS One 12:e169368 DOI: 10.1371/journal.pone.0169368.

378 Fisman DN, Abrutyn E, Spaude KA, Kim A, Kirchner C, Daley J. 2006. Prior pneumococcal vaccination is associated with reduced death, complications, and length of stay among hospitalized adults with community-acquired pneumonia. Clinical Infectious Diseases 42:1093-1101.

Haber M, Barskey A, Baughman W, Barker L, Whitney CG, Shaw KM, Orenstein W, Stephens DS. 2007. Herd immunity and pneumococcal conjugate vaccine: a quantitative model. Vaccine 25:5390-5398.

Johnstone J, Eurich DT, Minhas JK, Marrie TJ, Majumdar SR. 2010. Impact of the pneumococcal vaccine on long-term morbidity and mortality of adults at high risk for pneumonia. Clinical Infectious Diseases 51:15-22 DOI: 10.1086/653114.

Ministry of Health, Labour and Welfare, Japan. 2018a. Population census survey. Tokyo: Ministry of Health, Labour and Welfare. Available from: https://www.mhlw.go.jp/toukei/list/81-1a.html

Ministry of Health, Labour and Welfare, Japan. 2018b. Number of routine immunizations. Tokyo: Ministry of Health, Labour and Welfare. Available from: https://www.mhlw.go.jp/topics/bcg/other/5.html Miyashita N, Yamauchi Y. 2018. Bacterial pneumonia in elderly Japanese populations. Japanese Clinical Medicine 9:1179670717751433.

Naito T, Yokokawa H, Watanabe A. 2018. Impact of the national routine vaccination program on 23 -valent pneumococcal polysaccharide vaccine vaccination rates in elderly 
persons in Japan. Journal of Infection and Chemotherapy 24:496-498 DOI:

$399 \quad$ 10.1016/j.jiac.2018.01.004.

400 Omran AR. 2005. The epidemiologic transition: a theory of the epidemiology of population

401

402

403

404

405

406

407

408

409

410

411

412

413

414

415

416

417

418

419

420 change. Milbank Quarterly 83:731-757.

Ortqvist A, Hedlund J, Burman LA, Elbel E, Höfer M, Leinonen M, Lindblad I, Sundelöf B, Kalin M. 1998. Randomised trial of 23-valent pneumococcal capsular polysaccharide vaccine in prevention of pneumonia in middle-aged and elderly people. Swedish Pneumococcal Vaccination Study Group. Lancet 351:399-403.

Regev-Yochay G, Katzir M, Strahilevitz J, Rahav G, Finn T, Miron D, Maor Y, Chazan B, Schindler Y, Dagan R; IAIPD group. 2017. The herd effects of infant PCV7/PCV13 sequential implementation on adult invasive pneumococcal disease, six years post implementation: a nationwide study in Israel. Vaccine 35:2449-2456 DOI: 10.1016/j.vaccine.2017.03.031.

Simonsen L, Taylor RJ, Schuck-Paim C, Lustig R, Haber M, Klugman KP. 2014. Effect of 13-valent pneumococcal conjugate vaccine on admissions to hospital 2 years after its introduction in the USA: a time series analysis. Lancet Respiratory Medicine 2:387-394 DOI: $10.1016 / \mathrm{S} 2213-2600(14) 70032-3$.

Simonsen L, Taylor RJ, Young-Xu Y, Haber M, May L, Klugman KP. 2011. Impact of pneumococcal conjugate vaccination of infants on pneumonia and influenza hospitalization and mortality in all age groups in the United States. MBio 2:e00309-10 DOI: $10.1128 / \mathrm{mBio} .00309-10$.

The Japanese Respiratory Society. 2017. The JRS guidelines for the management of pneumonia in adults. Tokyo, Japan: Medical Review 1:2-8. 
421

422

423

424

425

426

427 Figure legends

428

429

430

431

432

442 the control. The vertical axis measures the causal effect that is calculated by $\beta$ (Year-2013)

Tsaban G, Ben-Shimol S. 2017. Indirect (herd) protection, following pneumococcal conjugated vaccines introduction: A systematic review of the literature. Vaccine 35:28822891 DOI: 10.1016/j.vaccine.2017.04.032.

Wing C, Simon K, Bello-Gomez RA. 2018. Designing difference in difference studies: best practices for public health policy research. Annual Review of Public Health 39:453-469 DOI: 10.1146/annurev-publhealth-040617-013507.

\section{Figure 1. Mortality trends of major causes of death in Japan, 1947-2017.}

A) Long-term mortality trends for the four leading causes of death in Japan, i.e., malignant neoplasm, heart disease, cerebrovascular disease, and pneumonia, are shown along with the longterm trend for tuberculosis mortality, which has substantially decreased since 1947. B) Enlarged view of recent mortality trends for three major causes of death, i.e., malignant neoplasm, heart disease, and pneumonia, that mostly maintain a parallel monotonic increasing trend. In 2009, the subsidized immunization with pneumococcal conjugate vaccine (PCV) was initiated in a limited number of municipalities. In 2014, routine immunization with PCV began, targeting those who newly reached $65,70,75,80,85,90,95$, or 100 years of age in the corresponding year from 2014-2018. The routine pneumococcal immunization of children started in 2013.

\section{Figure 2. Causal effect of the pneumococcal vaccine as estimated from prefectural} mortality data.

A-D) The causal effect of the pneumococcal vaccine based on an analysis including (A-B) or excluding (C-D) the 2017 data and using malignant neoplasm (A, C) or heart disease (B, D) as 
443 described from equation (1), where $\beta$ is the yearly reduction in the pneumonia mortality per

444100,000 individuals. The solid line shows the estimated causal effect, while the dashed and dash-

445 dotted lines show the $95 \%$ upper and lower confidence intervals, respectively.

446 Figure 3. Comparison of observed and predicted mortality from the analysis of

447 prefectural data.

448 A-B) Mortality calculated using malignant neoplasm (A) or heart disease (B) as the control

449 group. Grey circles represent the predicted mortality of malignant neoplasm (A) or heart disease

450 (B), squares show the median of malignant neoplasm (A) or heart disease (B) mortality among

451 the 47 prefectures, and error bars indicate the minimum and maximum values. The vertical axis

452 represents the mortality per 100,000 individuals. Black circles represent the predicted pneumonia

453 mortality, triangles show the median of pneumonia mortality among the 47 prefectures, and error

454 bars indicate the minimum and maximum values. The arrow indicates the year 2013 in which

455 routine immunization started among elderly.

456 Figure 4. Age- and gender-specific causal effect of pneumococcal vaccination.

457 A-D) The age- and gender-specific causal effect of the pneumococcal vaccine based on an

458 analysis including (A-B) or excluding (C-D) the 2017 data and using malignant neoplasm (A,

459 C) or heart disease (B, D) as the control. The causal effect in decreasing pneumonia mortality is

460 marked as a dashed line, and the $95 \%$ confidence intervals of the causal effect are presented

461 using error bars.

462 Figure 5. Comparison of observed and predicted age- and gender-specific mortality.

463 A-B) Age-stratified pneumonia mortality in males (A) or females (B), using malignant neoplasm

464 as the control for calculating predicted mortality. Each line represents the modelled (predicted) 
465 mortality. Each sign represents the observed mortality data. Since 2014, the year routine

466 immunization was introduced, the observed pneumonia mortality shows a decreasing trend. C-

467 D) Malignant neoplasm mortality by age in males (C) and females (D). E-F) Age-stratified

468 pneumonia mortality in males (E) or females (F), using heart disease as the control for

469 calculating predicted mortality. G-H) Heart disease mortality by age in males (G) or females

$470(\mathrm{H})$.

471

472 Supplementary Figure S1. Comparison of observed and predicted mortality of pneumonia and chronic obstructive pulmonary disease (COPD) from 2003-2017, Japan.

474 Predicted mortality for the entire Japan was calculated, using the COPD mortality as the control

475 group. The vertical axis represents the mortality per 100,000 individuals. Each line represents the

476 predicted mortality, while each mark represents the observed data.

477 


\section{Tables}

479 Table 1. Summary statistics of mortality by cause of death, gender and age

\begin{tabular}{|c|c|c|c|c|c|c|c|c|}
\hline \multirow[b]{2}{*}{ Disease } & \multirow[b]{2}{*}{ Gender } & \multirow[b]{2}{*}{ Age (years) } & \multicolumn{3}{|c|}{ With 2017} & \multicolumn{3}{|c|}{ Without 2017} \\
\hline & & & Min & Average & Max & Min & Average & Max \\
\hline malignant neoplasm & male & 65-69 & 618 & 680 & 768 & 622 & 685 & 768 \\
\hline malignant neoplasm & male & $70-74$ & 926 & 1036 & 1217 & 946 & 1044 & 1217 \\
\hline malignant neoplasm & male & $75-79$ & 1292 & 1519 & 1695 & 1307 & 1535 & 1695 \\
\hline malignant neoplasm & male & $80-84$ & 1925 & 2141 & 2242 & 1953 & 2157 & 2242 \\
\hline malignant neoplasm & male & 85-89 & 2723 & 2834 & 2933 & 2784 & 2842 & 2933 \\
\hline malignant neoplasm & male & $90 \&$ over & 3309 & 3431 & 3603 & 3309 & 3430 & 3603 \\
\hline malignant neoplasm & female & $65-69$ & 286 & 297 & 315 & 286 & 297 & 315 \\
\hline malignant neoplasm & female & $70-74$ & 388 & 421 & 458 & 395 & 424 & 458 \\
\hline malignant neoplasm & female & $75-79$ & 546 & 613 & 659 & 567 & 618 & 659 \\
\hline malignant neoplasm & female & $80-84$ & 842 & 906 & 965 & 863 & 910 & 965 \\
\hline malignant neoplasm & female & $85-89$ & 1217 & 1297 & 1387 & 1237 & 1303 & 1387 \\
\hline malignant neoplasm & female & $90 \&$ over & 1648 & 1691 & 1752 & 1648 & 1692 & 1752 \\
\hline heart disease & male & $65-69$ & 168 & 190 & 217 & 168 & 191 & 217 \\
\hline heart disease & male & $70-74$ & 253 & 297 & 357 & 259 & 300 & 357 \\
\hline heart disease & male & $75-79$ & 425 & 518 & 612 & 425 & 524 & 612 \\
\hline heart disease & male & $80-84$ & 814 & 964 & 1083 & 822 & 975 & 1083 \\
\hline heart disease & male & $85-89$ & 1623 & 1823 & 2082 & 1623 & 1837 & 2082 \\
\hline heart disease & male & $90 \&$ over & 3407 & 3664 & 3935 & 3407 & 3677 & 3935 \\
\hline heart disease & female & $65-69$ & 53 & 69 & 83 & 53 & 677 & 83 \\
\hline heart disease & female & $70-74$ & 100 & 1374 & 172 & 108 & 136 & 172 \\
\hline heart disease & female & $75-79$ & 2185 & 287 & 356 & 225 & 292 & 356 \\
\hline heart disease & female & $80-84$ & 508 & 640 & 759 & 514 & 649 & 759 \\
\hline heart disease & female & $85-89$ & 1167 & 1396 & 1624 & 1167 & 1411 & 1624 \\
\hline heart disease & female & $90 \&$ over & 3071 & 3317 & 3533 & 3071 & 3333 & 3533 \\
\hline pneumonia & male & 65-69 & 43 & 63 & 72 & 57 & 65 & 72 \\
\hline pneumonia & male & $70-74$ & 93 & 147 & 175 & 130 & 151 & 175 \\
\hline pneumonia & male & $75-79$ & 211 & 357 & 431 & 285 & 367 & 431 \\
\hline pneumonia & male & $80-84$ & 521 & 850 & 991 & 692 & 873 & 991 \\
\hline pneumonia & male & $85-89$ & 1231 & 1870 & 2199 & 1577 & 1916 & 2199 \\
\hline pneumonia & male & $90 \&$ over & 2883 & 4147 & 4764 & 3421 & 4237 & 4764 \\
\hline pneumonia & female & 65-69 & 11 & 19 & 23 & 16 & 19 & 23 \\
\hline pneumonia & female & $70-74$ & 24 & 46 & 58 & 39 & 47 & 58 \\
\hline pneumonia & female & $75-79$ & 67 & 120 & 149 & 94 & 123 & 149 \\
\hline pneumonia & female & $80-84$ & 187 & 320 & 389 & 252 & 330 & 389 \\
\hline pneumonia & female & $85-89$ & 490 & 805 & 978 & 630 & 828 & 978 \\
\hline pneumonia & female & $90 \&$ over & 1438 & 2176 & 2531 & 1744 & 2229 & 2531 \\
\hline
\end{tabular}

480 Mortality per 100,000 individuals is shown. Minimum and maximum refer to the largest and smallest yearly

481 mortality values from the year 2003 up to 2016 or 2017. 
483 Table 2. Estimates of the age-dependent causal effect parameter of pneumococcal

484 vaccination by age group and gender in Japan

\begin{tabular}{|c|c|c|c|c|c|c|c|c|}
\hline \multirow{3}{*}{$\begin{array}{l}\text { Age } \\
\text { (years) }\end{array}$} & \multicolumn{4}{|c|}{ With 2017 data } & \multicolumn{4}{|c|}{ Without 2017 data } \\
\hline & \multicolumn{2}{|c|}{ malignant neoplasm } & \multicolumn{2}{|c|}{ heart disease } & \multicolumn{2}{|c|}{ malignant neoplasm } & \multicolumn{2}{|c|}{ heart disease } \\
\hline & Male & Female & Male & Female & Male & Female & Male & Female \\
\hline $65-69$ & $\begin{array}{c}16 \\
(-28,59)\end{array}$ & $\begin{array}{c}1 \\
(-19,21)\end{array}$ & $\begin{array}{c}2 \\
(-37,41)\end{array}$ & $\begin{array}{c}3 \\
(-22,27)\end{array}$ & $\begin{array}{c}22 \\
(-42,86)\end{array}$ & $\begin{array}{c}3 \\
(-27,32)\end{array}$ & $\begin{array}{c}6 \\
(-50,63)\end{array}$ & $\begin{array}{c}5 \\
(-30,40)\end{array}$ \\
\hline $70-74$ & $\begin{array}{c}23 \\
(-20,67)\end{array}$ & $\begin{array}{c}5 \\
(-15,24)\end{array}$ & $\begin{array}{c}2 \\
(-37,41)\end{array}$ & $\begin{array}{c}5 \\
(-19,30)\end{array}$ & $\begin{array}{c}34 \\
(-30,98)\end{array}$ & $\begin{array}{c}8 \\
(-22,37)\end{array}$ & $\begin{array}{c}9 \\
(-47,66)\end{array}$ & $\begin{array}{c}9 \\
(-26,44)\end{array}$ \\
\hline $75-79$ & $\begin{array}{c}33 \\
(-11,75)\end{array}$ & $\begin{array}{c}3 \\
(-16,23)\end{array}$ & $\begin{array}{c}-9 \\
(-48,30)\end{array}$ & $\begin{array}{c}8 \\
(-16,33)\end{array}$ & $\begin{array}{c}53 \\
(-11,117)\end{array}$ & $\begin{array}{c}8 \\
(-21,37)\end{array}$ & $\begin{array}{c}4 \\
(-53,60)\end{array}$ & $\begin{array}{c}16 \\
(-19,51)\end{array}$ \\
\hline $80-84$ & $\begin{array}{c}-31 \\
(-74,12)\end{array}$ & $\begin{array}{c}-18 \\
(-38,2)\end{array}$ & $\begin{array}{c}-42 \\
(-81,-3)\end{array}$ & $\begin{array}{c}7 \\
(-18,31)\end{array}$ & $\begin{array}{c}-8 \\
(-72,56)\end{array}$ & $\begin{array}{c}-11 \\
(-41,18)\end{array}$ & $\begin{array}{c}-20 \\
(-76,36)\end{array}$ & $\begin{array}{c}21 \\
(-14,56)\end{array}$ \\
\hline $85-89$ & $\begin{array}{c}-143 \\
(-186,-99)\end{array}$ & $\begin{array}{c}-60 \\
(-80,-40)\end{array}$ & $\begin{array}{c}-98 \\
(-137,-59)\end{array}$ & $\begin{array}{c}-12 \\
(-37,12)\end{array}$ & $\begin{array}{c}-116 \\
(-180,-52)\end{array}$ & $\begin{array}{l}-49 \\
(-79 \\
-20)\end{array}$ & $\begin{array}{c}-52 \\
(-108,4)\end{array}$ & $\begin{array}{c}12 \\
(-23,47)\end{array}$ \\
\hline $\begin{array}{l}90 \& \\
\text { over }\end{array}$ & $\begin{array}{l}-357 \\
(-401, \\
-314) \\
\end{array}$ & $\begin{array}{l}-199 \\
(-218, \\
-179) \\
\end{array}$ & $\begin{array}{l}-276 \\
(-315, \\
-237) \\
\end{array}$ & $\begin{array}{l}-137 \\
(-162, \\
-113) \\
\end{array}$ & $\begin{array}{l}-339 \\
(-403, \\
-275) \\
\end{array}$ & $\begin{array}{l}-186 \\
(-215 \\
-156) \\
\end{array}$ & $\begin{array}{l}-226 \\
(-282 \\
-170) \\
\end{array}$ & $\begin{array}{l}-105 \\
(-140, \\
-70) \\
\end{array}$ \\
\hline
\end{tabular}

485

486 Figures should be interpreted as the yearly rate of reduction in pneumonia mortality per 100,000

487 individuals. Malignant neoplasm and heart disease were used as control groups. Upper and lower

$48895 \%$ confidence intervals, derived from profile likelihood, are shown in parenthesis. 
490 Table 3. Estimates of the regression parameters for the causal effect model of

491 pneumococcal vaccination applied to prefectural data in Japan

\begin{tabular}{ccccc}
\hline & \multicolumn{2}{c}{ With 2017 data } & \multicolumn{2}{c}{ Without 2017 data } \\
\cline { 2 - 5 }$\alpha_{0}$ & malignant neoplasm & heart disease & malignant neoplasm & heart disease \\
\cline { 2 - 5 }$\alpha_{1}$ & $267(264,271)$ & $141(138,144)$ & $268(264,271)$ & $142(138,145)$ \\
$\beta$ & $4(3,4)$ & $3(3,4)$ & $4(3,4)$ & $3(3,4)$ \\
$\gamma$ & $-10(-13,-8)$ & $-8(-10,-6)$ & $-9(-12,-6)$ & $-5(-7,-2)$ \\
$\delta$ & $-187(-190,-183)$ & $-58(-61,-55)$ & $-187(-191,-184)$ & $-59(-62,-56)$ \\
\hline
\end{tabular}

492

493 Figures should be interpreted as the yearly rate of reduction in pneumonia mortality per 100,000

494 individuals. Malignant neoplasm and heart disease were used as control groups. Upper and lower $49595 \%$ confidence intervals, derived from profile likelihood, are shown in parenthesis.

496 


\section{Table 4. Estimates of the causal effect of pneumococcal vaccination on pneumonia death 498 by age group and gender.}

\begin{tabular}{|c|c|c|c|c|c|c|c|c|}
\hline \multirow{3}{*}{$\begin{array}{l}\text { Age } \\
\text { (years) }\end{array}$} & \multicolumn{4}{|c|}{ With 2017 data } & \multicolumn{4}{|c|}{ Without 2017 data } \\
\hline & \multicolumn{2}{|c|}{ malignant neoplasm } & \multicolumn{2}{|c|}{ heart disease } & \multicolumn{2}{|c|}{ malignant neoplasm } & \multicolumn{2}{|c|}{ heart disease } \\
\hline & Male & Female & Male & Female & Male & Female & Male & Female \\
\hline $65-69$ & $20(-52,92)$ & $1(-32,34)$ & $2(-64,68)$ & $3(-38,44)$ & $\begin{array}{c}34(-90 \\
158)\end{array}$ & $4(-53,62)$ & $\begin{array}{c}9(-104 \\
123)\end{array}$ & $7(-63,77)$ \\
\hline $70-74$ & $24(-48,96)$ & $4(-29,37)$ & $0(-66,67)$ & $6(-35,47)$ & $\begin{array}{c}41(-83 \\
165)\end{array}$ & $8(-49,66)$ & $\begin{array}{c}13(-101 \\
126)\end{array}$ & $\begin{array}{c}12(-58 \\
82)\end{array}$ \\
\hline $75-79$ & $\begin{array}{c}42(-30 \\
114)\end{array}$ & $5(-29,38)$ & $\begin{array}{c}-13 \\
(-79,53)\end{array}$ & $\begin{array}{c}11(-30 \\
52)\end{array}$ & $\begin{array}{c}84(-40 \\
208)\end{array}$ & $\begin{array}{c}13(-45 \\
70)\end{array}$ & $\begin{array}{c}8(-106 \\
121)\end{array}$ & $\begin{array}{c}24(-46 \\
94)\end{array}$ \\
\hline $80-84$ & $\begin{array}{c}-38(-110 \\
34)\end{array}$ & $-25(-58,8)$ & $\begin{array}{c}-57(-124, \\
9)\end{array}$ & $8(-33,49)$ & $\begin{array}{c}0(-124 \\
124)\end{array}$ & $\begin{array}{c}-15(-73 \\
42)\end{array}$ & $\begin{array}{c}-23(-137 \\
90)\end{array}$ & $\begin{array}{c}33(-37 \\
103)\end{array}$ \\
\hline $85-89$ & $\begin{array}{c}-195(-267,- \\
123)\end{array}$ & $\begin{array}{c}-84(-118,- \\
51)\end{array}$ & $\begin{array}{c}-140(-206, \\
-73)\end{array}$ & $\begin{array}{c}-18(-59, \\
23)\end{array}$ & $\begin{array}{c}-167 \\
(-291-43)\end{array}$ & $\begin{array}{c}-74(-133,- \\
17)\end{array}$ & $\begin{array}{c}-73(-186, \\
41)\end{array}$ & $\begin{array}{c}24(-46 \\
94)\end{array}$ \\
\hline $90 \&$ over & $\begin{array}{c}-491(-563,- \\
419)\end{array}$ & $\begin{array}{c}-275(-308, \\
-242)\end{array}$ & $\begin{array}{c}-376(-442, \\
-310)\end{array}$ & $\begin{array}{c}-183(-224, \\
-142)\end{array}$ & $\begin{array}{c}-510(-634, \\
-386)\end{array}$ & $\begin{array}{c}-283(-340, \\
-226)\end{array}$ & $\begin{array}{c}-322(-436, \\
-209)\end{array}$ & $\begin{array}{c}-146(- \\
216,-76)\end{array}$ \\
\hline Prefecture & $2(-$ & & -3( & $8,2)$ & 1( & $5,8)$ & $-6(-$ & $1,0)$ \\
\hline
\end{tabular}

499

500 Figures should be interpreted as the yearly rate of reduction in pneumonia mortality per 100,000

501 individuals. Malignant neoplasm and heart disease were used as control groups. Except for the

502 last row, age-dependent model results are shown, while the last row give estimates from

503 prefecture-dependent model. Upper and lower 95\% confidence intervals, derived from profile

504 likelihood, are shown in parenthesis. 


\section{Figure 1 (on next page)}

Figure 1. Mortality trends of major causes of death in Japan, 1947-2017.
A) Long-term mortality trends for the four leading causes of death in Japan, i.e., malignant neoplasm, heart disease, cerebrovascular disease, and pneumonia, are shown along with the long-term trend for tuberculosis mortality, which has substantially decreased since 1947. B) Enlarged view of recent mortality trends for three major causes of death, i.e., malignant neoplasm, heart disease, and pneumonia, that mostly maintain a parallel monotonic increasing trend. In 2009, the subsidized immunization with pneumococcal conjugate vaccine (PCV) was initiated in a limited number of municipalities. In 2014, routine immunization with PCV began, targeting those who newly reached $65,70,75,80,85,90,95$, or 100 years of age in the corresponding year from 2014-2018. The routine pneumococcal immunization of children started in 2013. 
Figure 2 (on next page)

Figure 2. Causal effect of the pneumococcal vaccine as estimated from prefectural mortality data.

A-D) The causal effect of the pneumococcal vaccine based on an analysis including (A-B) or excluding (C-D) the 2017 data and using malignant neoplasm (A, C) or heart disease (B, D) as the control. The vertical axis measures the causal effect that is calculated by $\beta$ (Year-2013) described from equation (1), where $\beta$ is the yearly reduction in the pneumonia mortality per 100,000 individuals. The solid line shows the estimated causal effect, while the dashed and dash-dotted lines show the 95\% upper and lower confidence intervals, respectively. 

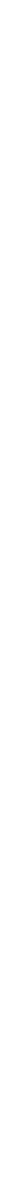


\section{Figure 3 (on next page)}

Figure 3. Comparison of observed and predicted mortality from the analysis of prefectural data.

A-B) Mortality calculated using malignant neoplasm (A) or heart disease (B) as the control group. Grey circles represent the predicted mortality of malignant neoplasm (A) or heart disease $(B)$, squares show the median of malignant neoplasm $(A)$ or heart disease $(B)$ mortality among the 47 prefectures, and error bars indicate the minimum and maximum values. The vertical axis represents the mortality per 100,000 individuals. Black circles represent the predicted pneumonia mortality, triangles show the median of pneumonia mortality among the 47 prefectures, and error bars indicate the minimum and maximum values. The arrow indicates the year 2013 in which routine immunization started among elderly. 

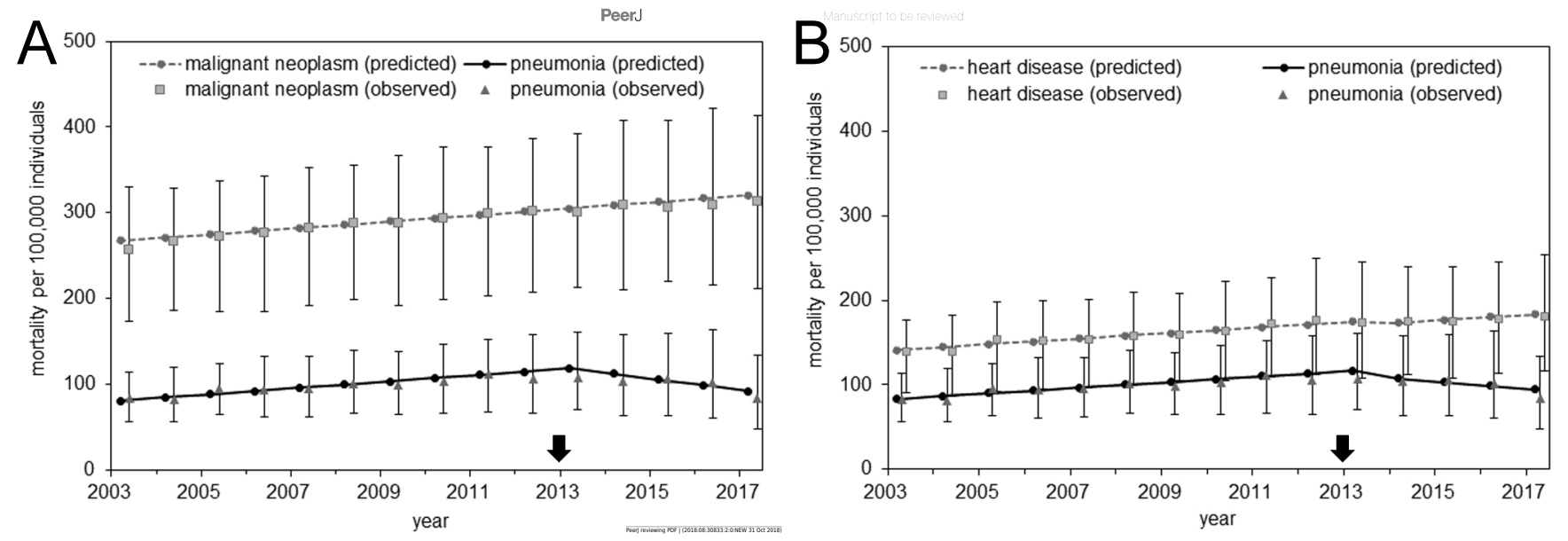
Figure 4 (on next page)

Figure 4. Age- and gender-specific causal effect of pneumococcal vaccination.

A-D) The age- and gender-specific causal effect of the pneumococcal vaccine based on an analysis including (A-B) or excluding (C-D) the 2017 data and using malignant neoplasm (A, C) or heart disease (B, D) as the control. The causal effect in decreasing pneumonia mortality is marked as a dashed line, and the $95 \%$ confidence intervals of the causal effect are presented using error bars. 


\section{Figure 5 (on next page)}

Figure 5. Comparison of observed and predicted age- and gender-specific mortality.

A-B) Age-stratified pneumonia mortality in males (A) or females (B), using malignant neoplasm as the control for calculating predicted mortality. Each line represents the modelled (predicted) mortality. Each sign represents the observed mortality data. Since 2014, the year routine immunization was introduced, the observed pneumonia mortality shows a decreasing trend. C-D) Malignant neoplasm mortality by age in males (C) and females (D). E-F) Age-stratified pneumonia mortality in males (E) or females (F), using heart disease as the control for calculating predicted mortality. G-H) Heart disease mortality by age in males $(G)$ or females $(H)$. 
